\title{
Proprioceptive Magnetic-Field Sensing for Closed-loop Control of Magnetic Capsule Endoscopes
}

\author{
Katie M. Miller, Arthur W. Mahoney, Thomas Schmid, and Jake J. Abbott
}

\begin{abstract}
The small intestine is the longest part of the gastrointestinal (GI) tract, but it is difficult to examine with traditional endoscopic procedures. To resolve this, swallowable capsules have been created that slowly pass through the entire GI tract taking images. Research is currently being done in magnetic actuation and localization to increase the speed and control of these smart pills. This paper focuses on a technique to more efficiently control a capsule endoscope based on proprioceptive sensing of the magnetic field. This sensing provides knowledge of the applied field in the frame of the capsule, and enables determination of its current state.
\end{abstract}

\section{INTRODUCTION}

Traditional endoscopic procedures such as colonoscopy and upper endoscopy are unable to examine the full length of the gastrointestinal (GI) tract. Wireless capsule endoscopy has become a useful diagnostic tool that enables clinicians to study this entire region and reduce patient discomfort. It consists of a swallowable capsule, containing a camera, which slowly moves through the GI tract taking images. These images are wirelessly transmitted to a receiver worn around the patient's waist. Several companies produce these "smart pills" [1]-[3], but they have two major limitations. First, they move through the GI tract passively with peristalsis, taking approximately eight hours to complete the examination. Second, the capsule randomly tumbles, often not taking quality photos and missing regions of interest.

Using a magnetic field to propel these capsules within the GI tract is a topic of considerable research. Numerous groups use an external rotating permanent magnet (PM) to apply magnetic forces and torques on a small PM placed inside the capsule. These forces and torques allow the capsule to be actuated in a desired direction and orientation [4][7]. The NORIKA capsule uses two sets of three induction coils, one internally and one externally, which interact to provide desired magnetic forces and torques [8]. Carpi et al. have used the Sterotaxis robotic navigation system originally designed for cardiovascular procedures to actuate a capsule endoscope through a pig's GI tract [9].

Regardless of the method of magnetic propulsion, the capsule must be localized within the patient to enable closedloop control. There are two popular methods for localization with magnetics. One strategy involves placing a small magnet

This work is supported by the National Science Foundation under grants 0952718 and 0654414.

K. M. Miller and A. W. Mahoney are with the School of Computing, T. Schmid is with the Department of Electrical and Computer Engineering, and J. J. Abbott is with the Department of Mechanical Engineering, University of Utah, Salt Lake City, UT 84112, USA. email: katie.millereutah.edu inside the capsule and using external sensors to find its position [10]-[12]. The other is to place sensors inside the capsule and localize relative to an external magnetic source. Guo et al. placed a tri-axial magnetic sensor inside a capsule for localization, but a PM was not included [13]. Lim et al. have used one-axis Hall effect sensors to detect the field at the center of a capsule, rather than the field at the center of their internal PMs [14]. They did not explain how saturation of the sensors was prevented from the close-proximity PMs. Salerno et al. placed a tri-axial sensor in a capsule adjacent to a PM, and used a wide-range sensor to prevent saturation [15].

This paper focuses on developing a magnetic sensor array to be placed inside of a capsule endoscope that measures the field at the center of the internal magnet without interference from the magnet itself. This sensor array could be used for localization in a non-uniform field, but we are implementing it for an alternative function: to determine the state of the capsule in a rotating magnetic field (e.g., Is the capsule's rotation synchronized with the rotating field, is it stuck, or is the capsule able to move but not remaining synchronized with the field?). We are interested in the field being experienced by the capsule, in its own frame of reference, hence our use of the term "proprioceptive." Knowledge of the capsule's state allows us to manipulate the externally applied magnetic field to control the capsule's motion, even without full localization.

To actuate our capsule, torque will be applied on the internal magnet from an external field, causing the capsule to rotate. In the future, a helix on the outer shell of a capsule will transfer rotational movement into forward motion [16]. The magnetic torque applied to the capsule, $\boldsymbol{\tau}=\mathbf{m} \times \mathbf{B}$, is maximized when the angle between the internal magnetic moment $\mathbf{m}$ and the magnetic field $\mathbf{B}$ is $90^{\circ}$. We will refer to this as the "lead angle." If we assume a linear model and that inertia is negligible, the magnetic torque is proportional to the angular velocity of the capsule through a damping coefficient $c$ as $|\boldsymbol{\tau}|=c \dot{\theta}$. Therefore, the maximum torque and speed occur simultaneously, when the lead angle is $90^{\circ}$. At higher angular velocities of the rotating field, the applied magnetic torque is no longer strong enough to enable the capsule to remain synchronized. This phenomenon is called "step-out". The drag coefficient $c$ is not known and may change as the capsule moves through different regions of the GI tract, so the desired angular speed cannot simply be calculated. However, if the lead angle is known, we can implement a close-loop control system to drive the lead angle to its ideal value of $90^{\circ}$, or any other desired value. 


\section{Sensor Layout Theory}

To measure the three-dimensional magnetic field vector inside the capsule, we chose to use six one-axis linear Hall effect sensors because each sensor only measures a magnetic field perpendicular to its surface; all other field components are ignored. This makes it possible to strategically place the sensors to prevent measurement of the capsule PM's field by orienting them so their measuring direction is locally orthogonal to the field. To provide a three-axis measurement of the externally applied field, each sensor is aligned so it measures one of the three cardinal directions. The propulsion of the capsule results from magnetic forces and torques exerted on the capsule's internal PM from the external field. If the field at the center of the internal PM is known, these forces and torques can be calculated and varied to control the behavior of the capsule. It is impractical to physically measure the field at the center of the magnet. However, because a magnetic field is continuous we can assume that the field is locally linear. This enables an accurate estimate of the applied field at the center by averaging two sensor readings with the same offset on either side of the magnet. Using this concept, we designed a sensor layout with two sensors in each axis to approximate the three-dimensional field at the magnet's center.

\section{A. Sensor Placement}

With the size constraints associated with capsule endoscopy and our assumption of a locally linear externally applied field, the sensors need to be in close proximity to the internal PM. To prevent saturation of a Hall effect sensor, its position needs to be located with little or no biasing field in the direction that it measures. Assuming the magnet can be accurately modeled using the point dipole model, the field $\mathbf{B}$, at a possible sensor position $\mathbf{p}$, can be calculated using:

$$
\mathbf{B}(\mathbf{p})=\frac{\mu_{0}}{4 \pi|\mathbf{p}|^{3}}\left(\frac{3(\mathbf{m} \cdot \mathbf{p}) \mathbf{p}}{|\mathbf{p}|^{2}}-\mathbf{m}\right)
$$

where $\mathbf{m}$ is the magnetic moment of the magnet and $\mu_{0}$ is the permeability of free space [17].

The field of a point dipole, as illustrated in Fig. 1, has positions where the magnetic field points in one cardinal direction. A sensor can be placed in one of these positions such that its surface is parallel to the field of the internal PM to prevent interference in the measurements. From Fig. 1, a sensor measuring the applied magnetic field in the $\mathbf{y}$ direction should not be placed in a region labeled with $\mathbf{B}_{\mathbf{y}}$ because the field is only in that direction and the sensor will be saturated by the internal PM's field. Instead, the sensor should be placed in any region labeled with $\mathbf{B}_{\mathbf{z}}$. The magnetic field is radially symmetric, so the sensors measuring the applied field in the $\mathbf{x}$ direction can be placed in an analogous position to the $\mathbf{y}$ sensors, but along the $\mathbf{x}$ axis.

The sensors for the $\mathbf{z}$ direction are more difficult to place. The only positions in the dipole field (Fig. 1) with no component in the $\mathbf{z}$-axis exist on the peak of the dipole field curve. At these locations the field lies solely in the horizontal $\mathbf{x}-\mathbf{y}$ plane. These positions lie along a line drawn out from

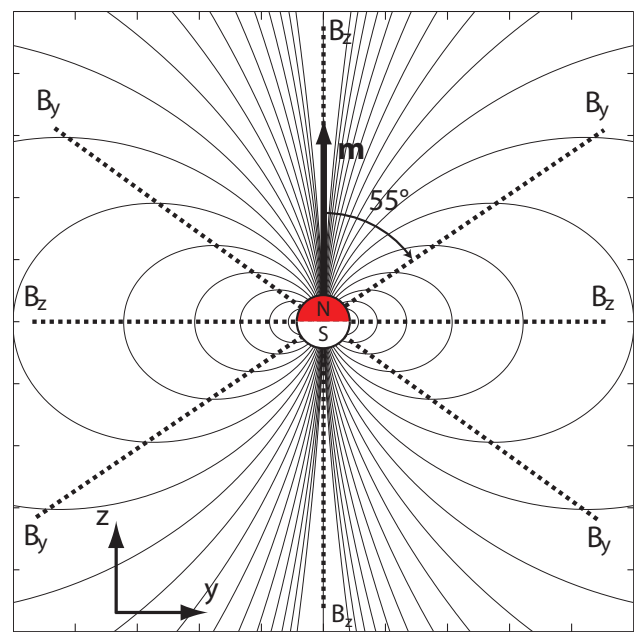

Fig. 1. The magnetic field using a point dipole model has regions where the field is in only one cardinal direction. These regions, denoted by the dashed lines, are the possible locations for our sensors. Accompanying each dashed line is the field direction that a sensor should not measure from that position because its measurements will be corrupted by the internal magnet.

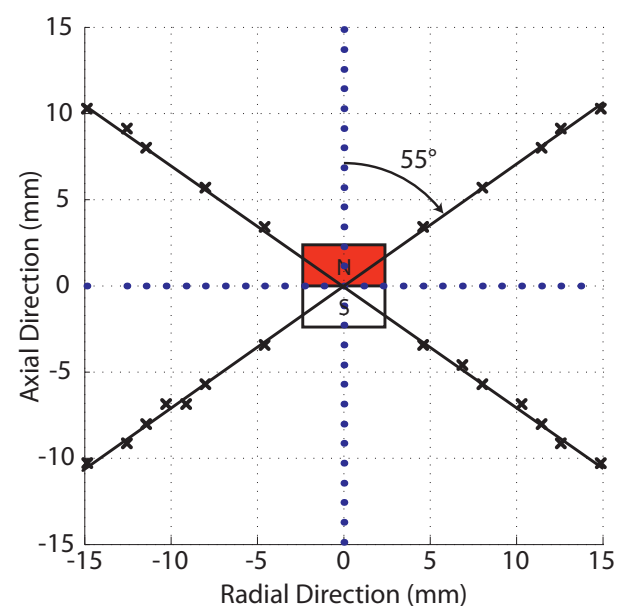

Fig. 2. The field of a $108 \mathrm{~mm}^{3}$ cubic magnet was calculated using Maxwell FEA software. This plot shows where the magnetic field is either completely vertical (blue dots) or completely horizontal (black Xs) along with lines showing the dipole model's prediction.

the middle of the magnet at an angle of approximately $55^{\circ}$ measured from $\mathbf{m}$.

The cubic magnet we chose can not be perfectly modeled by the point dipole in the close region where the sensors are placed. To confirm locations where the magnetic field would be zero in each axis, we plotted the field of our chosen $108 \mathrm{~mm}^{3}$ cubic magnet using Ansoft Maxwell version 14.0 FEA software. Figure 2 depicts the field where either the radial or axial portion of the magnetic field is zero. Our predicted regions using the point dipole model were a close approximation as shown. We determined the positions for the six sensors, two in each axis, by placing them at points where the software predicted the field to be minimal. The final layout is illustrated in Fig. 3. 


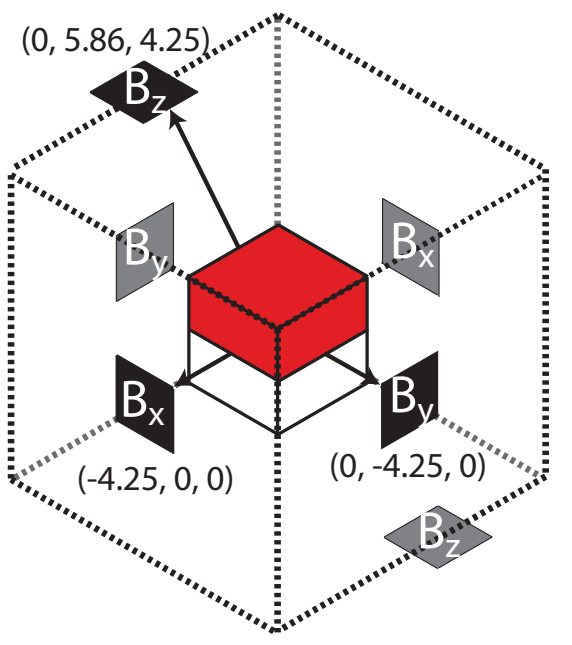

Fig. 3. An isometric view of our layout with each sensor labeled with the field direction it is measuring, and its final position in $\mathrm{mm}$ with respect to the center of the magnet. The gray sensors are on the backside of the cube and are not visible from this angle, but are located at the negative counterpart of the corresponding sensor. This view has been chosen to clearly show the positioning of all six sensors.

\section{Prototype CApsule}

A prototype capsule was created that measures the magnetic field at the center of the capsule's PM and wirelessly transmits the data to a computer. Using the information described in the previous section, we created a cubic sensor array with a Hall effect sensor placed on each side of a cube resulting in two Hall effect sensors measuring each of the three orthogonal axes. Allegro A1392 linear Hall effect sensors with a sensitivity of $25 \mathrm{~V} / \mathrm{T}$ and range of $\pm 64 \mathrm{mT}$ were chosen. In the radial axes, the sensor is placed at $4.25 \mathrm{~mm}$ measured from the middle of the magnet along the axis. We chose the point $(5.86 \mathrm{~mm}, 4.25 \mathrm{~mm})$ along the radial and axial directions respectively as the center point for the Hall effect sensor in the $\mathbf{z}$ direction because this was the closest position to the magnet's center, with predicted minimal field, that could be realized with the size constraints of our chosen components. The final Hall effect sensor was placed at the negative counterpart. Using this configuration, the maximum DC offset from the internal PM was $8.63 \mathrm{mT}$, which was from a $\mathbf{B}_{\mathbf{x}}$ sensor. This is a significant improvement over the alternative of placing the $\mathbf{B}_{\mathbf{z}}$ sensor along the $\mathbf{z}$-axis at $4.25 \mathrm{~mm}$ similar to the $\mathbf{x}$ and $\mathbf{y}$ sensors. The field at this position was measured by a Hirst GM08 gaussmeter as approximately $250 \mathrm{mT}$.

The cubic sensor array was designed with six parts shaped similar to jigsaw puzzle pieces. This design was chosen for two major reasons. First, the pieces fit securely together around the $108 \mathrm{~mm}^{3}$ Grade-N52 NdFeB PM. The jigsaw shape ensures that the sensors remain perpendicular to each other and pointed along the desired axes they are measuring. Second, the shape helps to keep the sensor array rigidly in place as the capsule rotates because the protruding edges are placed into press-fit slots. The sensor array is pictured

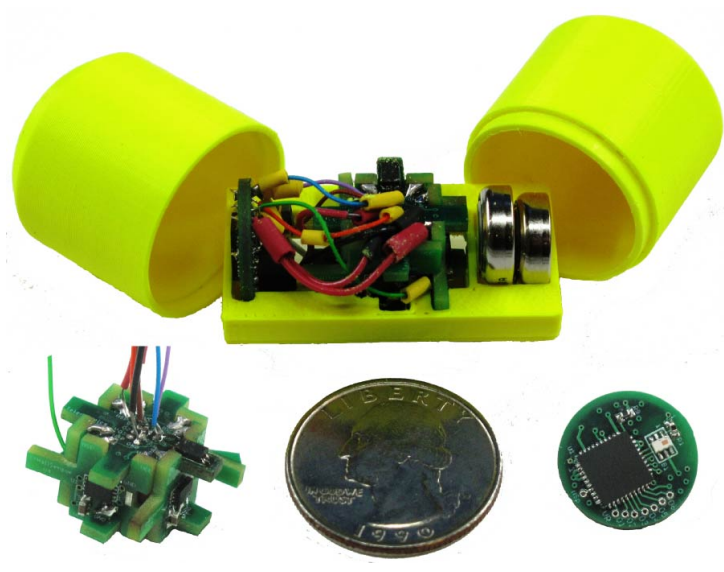

Fig. 4. The capsule contains, from left to right, the communications electronics including the microcontroller and transceiver, the Hall effect sensor array surrounding a PM, and the coin-cell batteries. The sensor array and PCB for wireless communication are shown on the bottom left and right, respectively, for a closer view.

on the left of Fig. 4. Also shown in Fig. 4 on the right is the PCB used for wireless communication. A Texas Instruments CC2530 microcontroller was chosen because of its small size, low power consumption, and its integrated Zigbee transceiver. This two-sided circular board contains the necessary hardware components to read the sensor values, convert them to a digital signal, and then transmit the data wirelessly to another CC2530 microcontroller attached to a personal computer through a serial port. These electronic boards were enclosed along with two $1.55 \mathrm{~V}$ Energizer coincell batteries inside a rapid-prototype capsule as shown in Fig. 4. For easy access to the electronics and batteries, the capsule was designed as two halves that fit together. A rack, which has press-fit slots for every component, slides into each half of the capsule to ensure all parts remain stationary as the capsule rotates. The dimensions of the assembled capsule are $50.5 \mathrm{~mm}$ in length by $25 \mathrm{~mm}$ in diameter. When scaling this design to the commercially available size of approximately $26 \mathrm{~mm}$ by $11 \mathrm{~mm}$ it is important to note that the relative positioning of the sensors will remain the same.

\section{EXPERIMENTAL Setup}

To test our hypothesis of using the magnetic sensors' readings for improved control, we constructed an experimental setup as shown in Fig. 5. A set of Helmholtz coils were used because they provide a uniform magnetic field at the center in any direction. However, the envisioned final system will utilize a single rotating permanent magnet mounted on a 6-DOF robotic arm because of its scalability for clinical use [5]. The capsule was rotated inside of a clear acrylic tube with an inner diameter of $37 \mathrm{~mm}$ that was inserted into the center of the coils. Lubricant was used to minimize the effects of friction, and rigid rods pinned the tube in place to prevent movement as the capsule rotated. Each sensor was calibrated before every test to remove small offsets by averaging 100 measurements with no magnetic field present.

The capsule was tested in three conditions. First, it was 


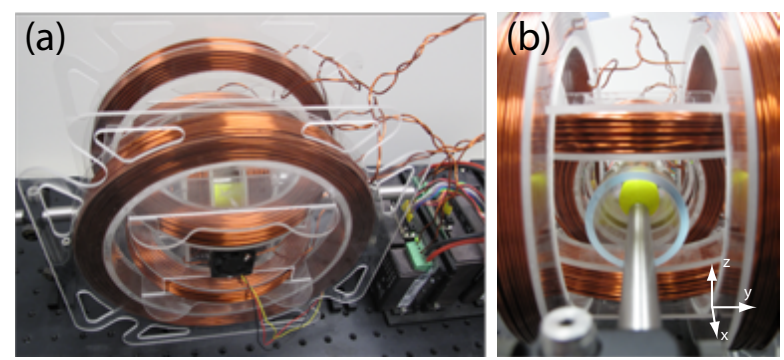

Fig. 5. (a) An overall view of the experimental setup with the Helmholtz coils. (b) A side view into the Helmholtz coils with the capsule inserted in an acrylic tube. The rod holds the tube in place as the capsule rotates.

held in place to simulate being stuck with the field rotating around it in the $\mathbf{y}-\mathbf{z}$ plane. Second, the capsule rotated with the magnetic field. Finally, the field rotated above the stepout frequency. The results are discussed in the following section. The sensors are sampled at a rate of $20 \mathrm{~Hz}$ and the data is wirelessly transmitted to a computer. All calculations are performed online, enabling closed-loop control.

\section{SEnsor Results And Discussion}

We started by holding the capsule stationary using two rigid rods in a rotating magnetic field with a frequency of $0.5 \mathrm{~Hz}$ and magnitude of $6.75 \mathrm{mT}$. As demonstrated in Fig. 6(a), the field magnitude oscillates between approximately $\pm 6.5 \mathrm{mT}$ with a phase shift between the $\mathbf{y}$ and $\mathbf{z}$ axis of $90^{\circ}$, which occurs because the sensors are orthogonal. The field rotates completely around the capsule so the lead angle changes $360^{\circ}$ or $\pm 180^{\circ}$ as shown in the bottom portion of Fig. 6(a). The period is 2 seconds, which is anticipated with our $0.5 \mathrm{~Hz}$ frequency. To further illustrate the sensor measurements, Fig. 6(b) shows the components of the $\mathbf{B}$ field along with the predicted field. The predictions were found by measuring 15 full rotations of the field at each of our chosen magnitudes with a Hirst GM08 gaussmeter and averaging the peak amplitude across the trials. The $\mathbf{x}$ component is almost zero because the field is rotating in the $\mathbf{y}-\mathbf{z}$ plane. The slight offset may be due to a small angle induced in the capsule orientation from the rods holding the capsule in place.

Next, the capsule was tested in a magnetic field with a magnitude of approximately $9.75 \mathrm{mT}$ rotating at $1 \mathrm{~Hz}$. The magnetic field that the sensors detect remains fairly constant because the capsule is rotating in sync with the field so it does not appear as though the field is rotating in the capsule's frame of reference, as shown in Fig. 6(c). A low-pass filter was implemented to minimize noise. The rotation of the capsule is $1 \mathrm{~Hz}$ so a cutoff frequency of $3 \mathrm{~Hz}$ was used to remove any high frequency changes in the transmitted data. Figure 6(d) further shows that the capsule experiences a field that only slightly oscillates because the measurements are only distributed over a small portion of the circle.

If the magnetic field is rotating too quickly for the capsule to align itself, or the magnitude of the field is not strong enough to rotate the capsule synchronously (these two cases are coupled), then the field is rotating faster than the capsule's step-out frequency, and the capsule rotates backward and forward chaotically trying to align itself with the field. This results in magnetic field oscillations similar to when the capsule was held fixed as demonstrated by Fig. 6(e). However, between two and three seconds the capsule is briefly able to rotate with the field, causing the field magnitude and lead angle to stay more constant before it steps out again. In step-out, the lead angle oscillates between a positive and negative value because the capsule rotates in both directions in an effort to align with the rotating field with no net progression. Fig. 6(f) shows that the capsule behaves similar to the stationary tests with the sensor measurements no longer as distinct points because the capsule can rotate. The capsule's outer diameter is significantly smaller than the inner diameter of the tube, and the erratic motion of the capsule induces lateral motion, which results in the scattering of the dots.

It is visually perceptible that the three cases in Fig. 6, (fully stuck, rotating synchronously, and stepped-out of synchronization) are distinguishable. Signal processing will enable future controllers to quickly and robustly diagnose the state of the capsule.

\section{Vi. Closed Loop Controller}

A simple PI controller was created to drive the lead angle to a desired value. To test it in an ideal environment with no friction or capsule vibration, the magnitude of the field was chosen such that it did not provide enough torque to rotate the capsule. Figure 7 demonstrates the controller's effectiveness at driving the capsule's lead angle from an unknown start state to an arbitrary angle of $60^{\circ}$ using data collected from our Hall effect sensors. After the angle converges to the desired value, it is possible to infer the magnet's orientation inside the coils because of the known magnitude and lead angle of the external field. This information could be used in the future for localization.

In a real-world setting, the velocity is a more likely choice of a desired parameter. An achievable angular speed can be met with a range of field magnitudes. However, a larger lead angle allows for a smaller field magnitude to provide the same torque, $|\boldsymbol{\tau}|=|\mathbf{m}||\mathbf{B}| \sin (\alpha)$, where $\alpha$ is the lead angle between $\mathbf{m}$ and $\mathbf{B}$. Algorithm 1 was designed to control the field magnitude to its lowest possible value. If the field is produced by an external PM, this would enable it to be farther from the patient while still rotating the capsule at the desired rotational velocity. The algorithm first decides if the capsule is in step-out by examining the sign of the lead angle for $n$ previous values of $\alpha$. This was chosen as half the period because the lead angle will change sign at least once during this duration if it is in step-out or stuck. When the capsule is rotating synchronously with the field, to prevent oscillations, the magnitude is held constant if the lead angle is within a desired tolerance (chosen as $\pm 5^{\circ}$ of the desired angle).

To demonstrate this controller, we chose to use a desired angle of $50^{\circ}$ to avoid the step-out region. As illustrated by Fig. 8, it successfully drives the magnitude to the lowest possible value and the lead angle converges to our desired angle. 

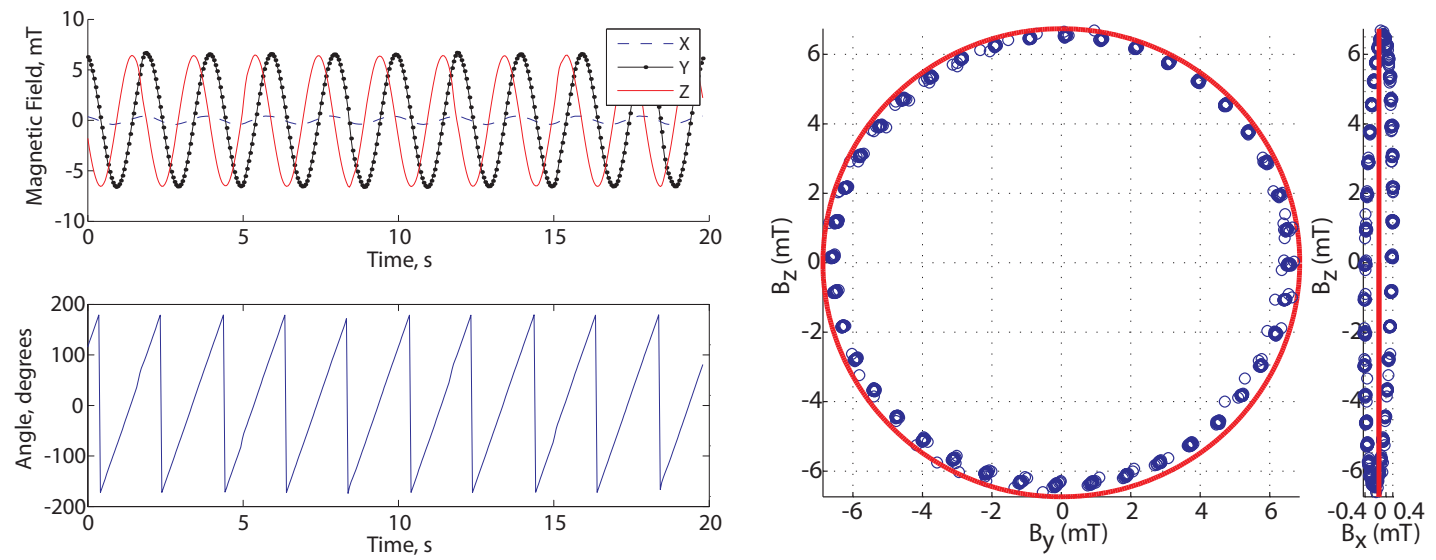

(a) Stationary capsule in a rotating field of $6.75 \mathrm{mT}$ at $0.5 \mathrm{~Hz}$

(b) Stationary capsule in a rotating field of $6.75 \mathrm{mT}$ at $0.5 \mathrm{~Hz}$.
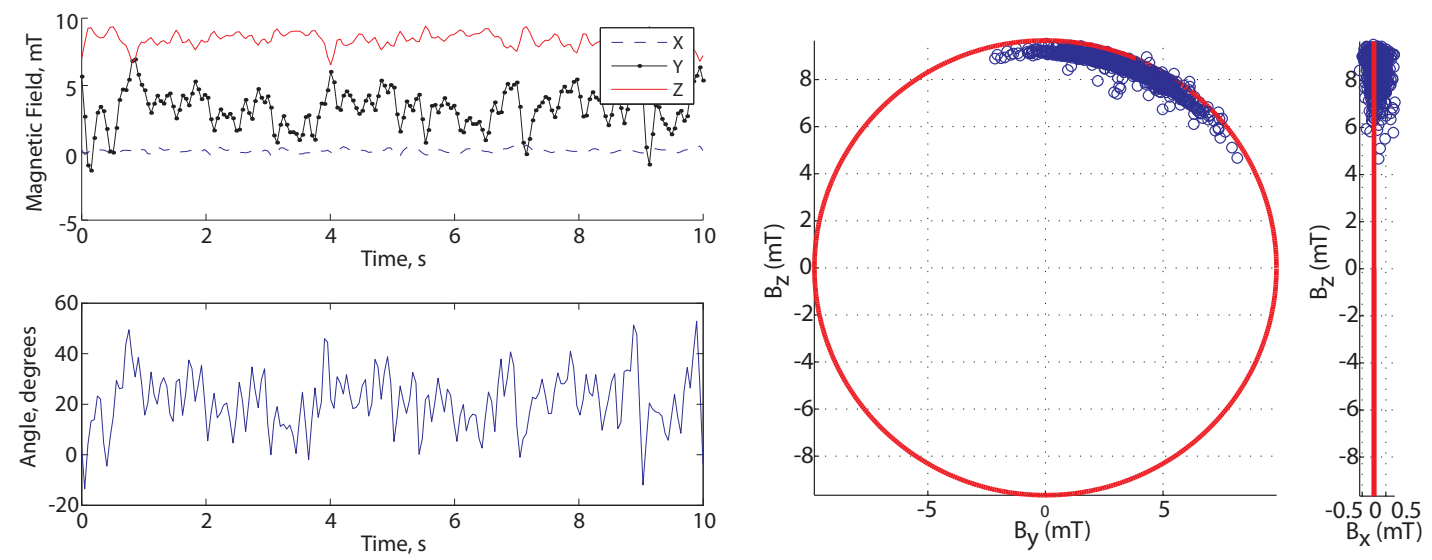

(c) Capsule rotating synchronously in a field of $9.75 \mathrm{mT}$ at $1 \mathrm{~Hz}$

(d) Capsule rotating synchronously in a field of $9.75 \mathrm{mT}$ at $1 \mathrm{~Hz}$.
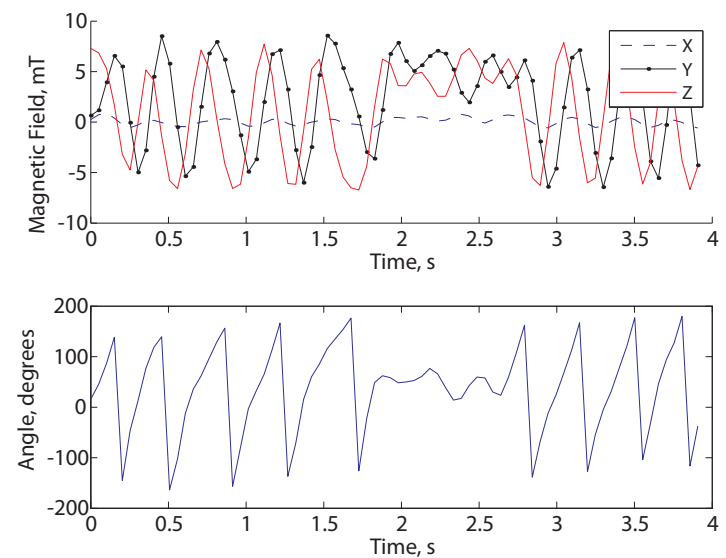

(e) Capsule stepping out in a field of $7.75 \mathrm{mT}$ at $3 \mathrm{~Hz}$.

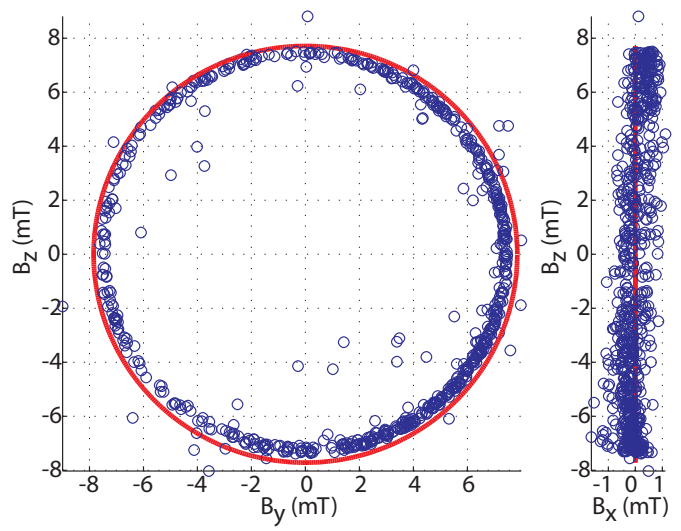

(f) Capsule stepping out in a field of $7.75 \mathrm{mT}$ at $3 \mathrm{~Hz}$.

Fig. 6. Graphs on the left show the measured magnetic field $\mathbf{B}$ and lead angle vs. time. On the right are the magnetic field components from the sensors overlaid on the predicted field. The field characteristics for each graph are in the subcaptions.

Even when the capsule goes into step-out at 11 seconds, the controller allows the capsule to quickly recover and align itself with the rotating field. Notice that the magnitude stays maximized for a few seconds after the step-out occurs; this is because the capsule is still considered to be in step-out until the lead angle has been positive for the chosen time frame of half a cycle. The duration of this time frame is directly dependent on the rotating field's frequency.

\section{CONCLUSION}

A sensor configuration was designed that enables a threedimensional magnetic field measurement at the center of the capsule's internal PM without interference from the magnet itself. It was experimentally shown that the data from these sensors provides the necessary information to distinguish 

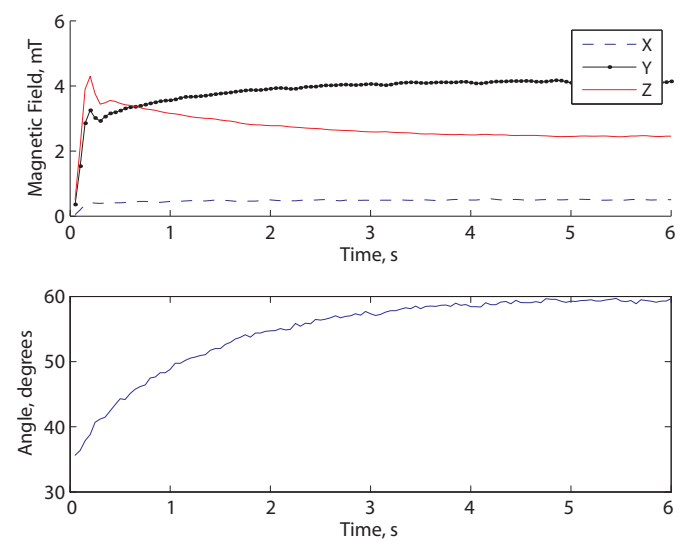

Fig. 7. The measured magnetic field $\mathbf{B}$ and lead angle vs. time. The lead angle is being controlled to a desired value of $60^{\circ}$ using a PI controller with a field magnitude set at $6 \mathrm{mT}$ such that the capsule does not rotate.

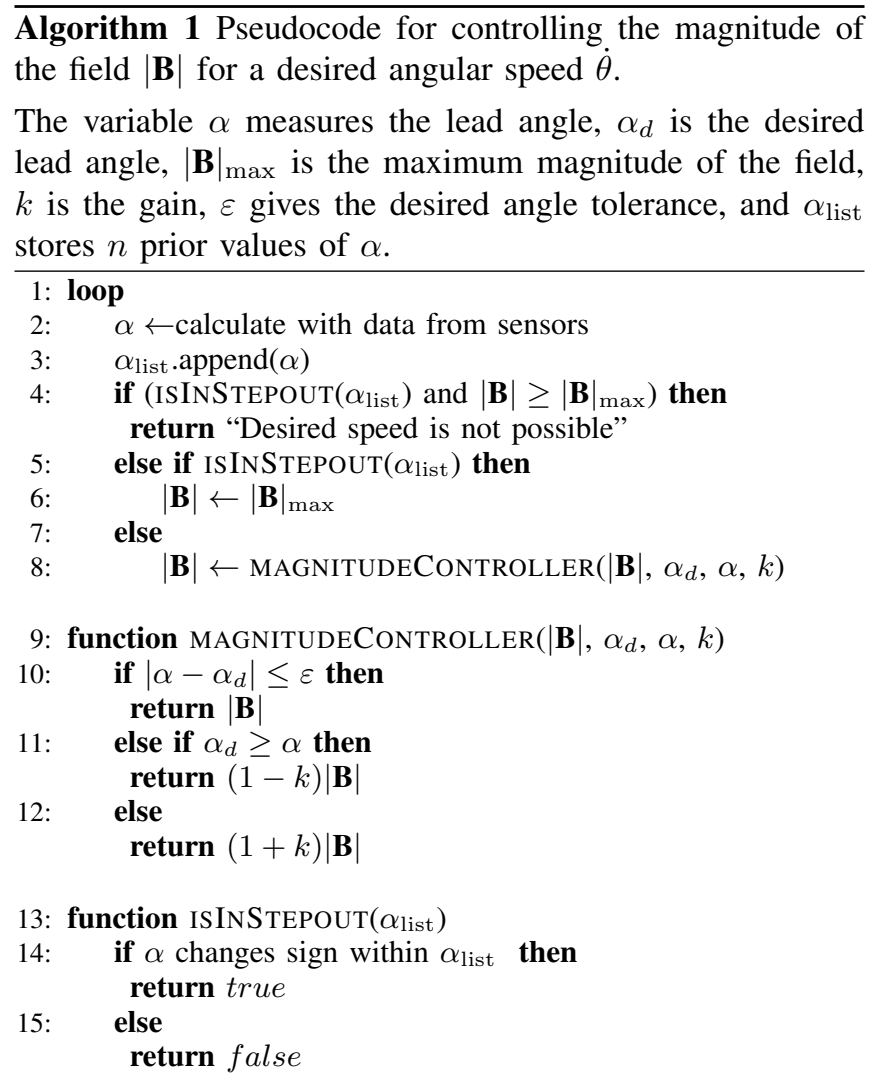

between the three possible capsule states: synchronous rotation, step-out, and stuck. A closed-loop controller was implemented to control the lead angle of the capsule to prevent step-out, while providing the smallest field magnitude that will enable the capsule to synchronously rotate at some desired speed. In the future, the control system can be extended to adapt the given desired speed to an attainable value if it is currently unreachable. The sensor arrangement can also be used to localize the capsule.

\section{REFERENCES}

[1] Given Imaging, http://www.givenimaging.com. PillCam, 2012.
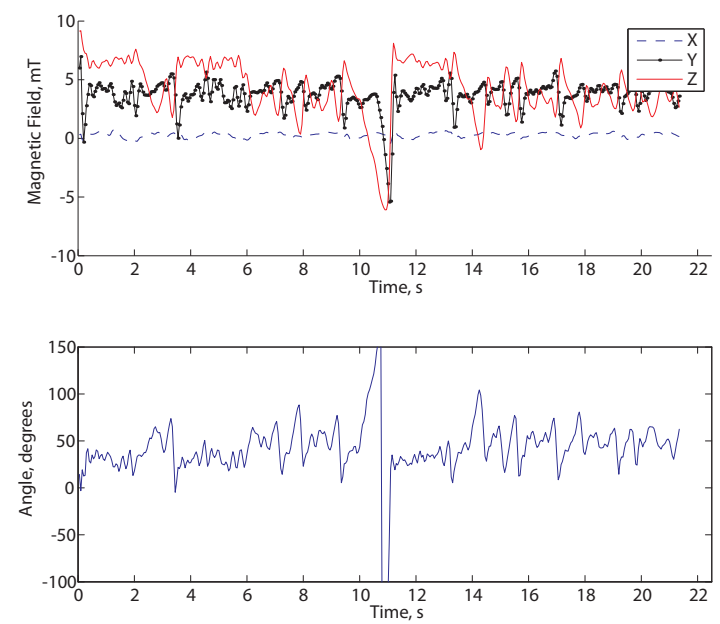

Fig. 8. The measured magnetic field $\mathbf{B}$ and lead angle vs. time. The controller converges to the lowest magnetic field strength possible to still rotate the capsule synchronously with a desired speed of $0.5 \mathrm{~Hz}$.

[2] Olympus. EndoCapsule. http://www.olympus-europa.com/medical, 2012.

[3] RF System, http://www.rfamerica.com/sayaka. Sayaka, 2012.

[4] G. Ciuti, P. Valdastri, A. Menciassi, and P. Dario. Robotic magnetic steering and locomotion of capsule endoscope for diagnostic and surgical endoluminal procedures. Robotica, 28:199-207, 2010.

[5] A.W. Mahoney, D. L. Cowan, K. M. Miller, and J.J. Abbott. Control of untethered magnetically actuated tools using a rotating permanent magnet in any position. In IEEE Int. Conf. Robot. Auto., pages 231234, 2012.

[6] X. Wang and M.Q.-H. Meng. Computational aspects in actuation and guidance mechanism for wireless active capsule endoscope. In IEEE Int. Conf. Intelligent Robots and Systems, pages 1198-1203, 2008.

[7] J. Kim, Y. Kwon, and Y. Hong. Automated alignment of rotating magnetic field for inducing a continuous spiral motion on a capsule endoscope with a twistable thread mechanism. Int. J. of Prec. Eng. \& Мапи., 13(3):371-377, 2012.

[8] A. Uehara and K. Hoshina. Capsule endoscope NORIKA system. Min Invas Ther and Allied Technol, 1(1):227-334, 2003.

[9] F. Carpi, N. Kastelein, M. Talcott, and C. Pappone. Magnetically controllable gastrointestinal steering of video capsules. IEEE Trans. Biomed. Eng., 58(2):231-234, 2011.

[10] C. Hu, M. Li, S. Song, W. Yang, R. Zhang, and M.Q.-H. Meng. A cubic 3-axis magnetic sensor array for wirelessly tracking magnet position and orientation. IEEE Sensors J, 10(5):903-913, 2010.

[11] W. He, G. Yan, and X. Guo. Analysis of magnetic marker localization method for non-invasive detecting capsule with batteries in GI tract. In IEEE Int. Conf Elec. Meas. Instr, pages 3-30-3-34, 2009.

[12] W. Yang, C. Hu, M.Q.-H. Meng, S. Song, and H. Dai. A sixdimensional magnetic localization algorithm for a rectangular magnet objective based on a particle swarm optimizer. IEEE Trans. Mag, 45(8):3092-3099, 2009.

[13] X. Guo, G. Yan, and W. He. A novel method of three-dimensional localization based on a neural network algorithm. J of Med Eng \& Tech, 33(3):192-198, 2009.

[14] M. Kim, Y. Hong, and E. Lim. Position and orientation detection of capsule endoscopes in spiral motion. Int. J of Prec. Eng. and Manu., 11(1):31-37, 2010.

[15] M. Salerno, G. Ciuti, G. Lucarini, R. Rizzo, P. Valdastri, A. Menciassi, A. Landi, and P. Dario. A discrete-time localization method for capsule endoscopy based on on-board magnetic sensing. Meas. Sci. Technol., 23(1):015701, 2012.

[16] M. Sendoh, K. Ishiyama, and K.-I.Arai. Fabrication of magnetic actuator for use in a capsule endoscope. IEEE Trans. Magnetics, 39(5):3232-3234, 2003.

[17] E. P. Furlani. Permanent magnet and electromechanical devices: materials, analysis, and applications. Academic Press, San Diego, California, 1st edition, 2001. 